\title{
Avoiding dacryocystorhinostomy in cases of epiphora caused by inferior meatus obstruction
}

\author{
Dvir Koenigstein $\mathbb{1}^{1} \cdot \operatorname{Ran}$ Ben Cnaan ${ }^{1} \cdot$ Shay Keren $\mathbb{1}^{1} \cdot$ Igal Leibovitch $^{1} \cdot$ Ahmad Safadi $^{2} \cdot$ Roee Landsberg $^{2} \cdot$ \\ Avraham Abergel ${ }^{2}$
}

Received: 18 October 2017 / Revised: 22 March 2018 / Accepted: 3 April 2018 / Published online: 18 May 2018

(c) The Royal College of Ophthalmologists 2018

\begin{abstract}
Aims To determine the role of inferior meatus pathologies as an underdiagnosed cause of epiphora.

Methods This study was conducted in the oculoplastic institution of Tel Aviv medical center-a regional referral center. A retrospective review of files of patients presenting to the lacrimal clinic with nasolacrimal duct obstruction between October 2010 and September 2016. Cases in which a pathology of the inferior meatus was identified and treated are presented in this article.

Results During this time frame, we preformed 186 endoscopic dacryocystorhinostomy surgeries. Out of those, eight patients (4.3\%) were diagnosed and treated for pathology causing an obstruction of the inferior meatus. Seven of our patients were females; the mean age was 24 years. A wide range of pathologies were found: cysts, dacryoliths, membranes obstructing the inferior meatus, and concheal obstruction. All patients went through endoscopic treatment targeted at the cause of obstruction. During follow-up (average 35 months) only two patients remained symptomatic and were referred for an endonasal endoscopic dacryocystorhinostomy.

Conclusions Inferior meatus obstruction is an underdiagnosed cause of epiphora. Multiple pathologies may co-exist in the same patient. In select cases of NLDO, diagnosis and treatment can be done endoscopically, avoiding the need for dacryocystorhinostomy.
\end{abstract}

\section{Introduction}

Nasolacrimal duct obstruction (NLDO) is a common cause of epiphora. The most common cause of primary NLDO is chronic inflammation and fibrosis [1]. Secondary causes of NLDO are less common and diagnosis may require a more extensive workup. Correct diagnosis of the obstruction site and its nature is essential for choosing a treatment plan and

These authors contributed equally: Dvir Koenigstein, Ran Ben Cnaan.

Dvir Koenigstein

Dvirkk@gmail.com

1 Oculoplastics Institute, Division of Ophthalmology, Tel Aviv Sourasky Medical Center Affiliated with the Sackler Faculty of Medicine, Tel Aviv University, Tel Aviv, Israel

2 Ear, Nose and Throat Department, Tel Aviv Sourasky Medical Center Affiliated to Sackler Faculty of Medicine, Tel Aviv University, Tel Aviv, Israel a surgical approach [2]. Recent reports suggest that obstructions of Hasner's valve (inferior meatus, IM) are significantly more common in adults than previously appreciated [3-6]. In this paper, we will describe a wide variety of pathologies of the IM that represent our experience and offer a diagnostic approach for future cases.

\section{Materials and methods}

This study was approved by the institutional Helsinki review board. Patients with symptomatic epiphora and a suspected NLDO were referred to our multi-disciplinary lacrimal clinic between October 2010 and September 2016. At the clinic, examination by an oculoplastic specialist and an otorhinolaryngology specialist were performed. Evaluation included slit-lamp microscopy, nasolacrimal syringing, and endoscopic evaluation of the nasal cavity. In selected cases the IM was visualized prior to surgery. In this manuscript we have included all patients who were diagnosed and treated for a pathology of the IM during this time 


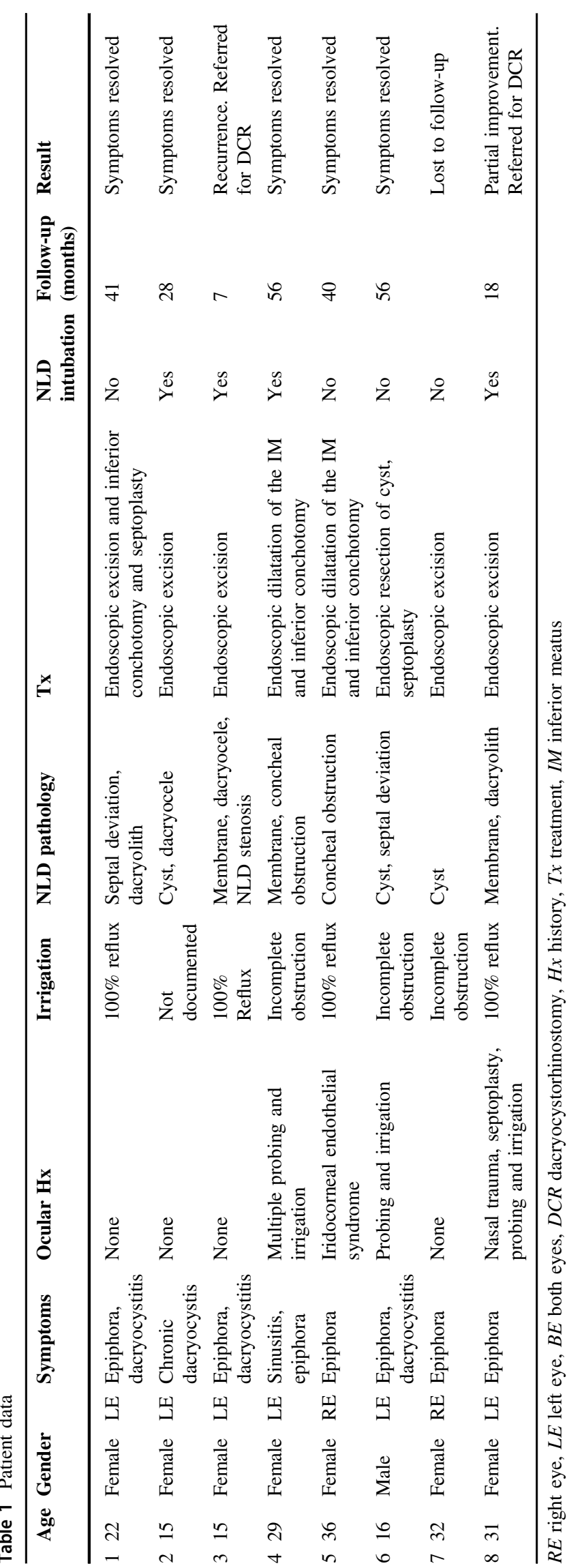

frame. Data gathered from the medical records were: age, sex, medical and ocular history, details from ocular and endoscopic examination. Patients' surgical records and imaging exams were also reviewed.

All patients underwent surgical intervention in the operating room under general anesthesia. Irrigation of the nasolacrimal duct (NLD) was performed using a 21-guage lacrimal cannula and a diluted fluorescein solution. We conducted an endoscopic evaluation of the nasal cavity and of the IM. Surgical procedure varied according to the pathology found in the IM. All surgeries were concluded with a repeated irrigation of the NLD demonstrating free passage to the nasal cavity. Follow-up was performed in the outpatient clinic. Patients also completed a telephonebased questionnaire assessing current symptoms of epiphora.

\section{Results}

During this time frame, we preformed 186 endoscopic DCR surgeries. Out of those, eight patients (4.3\%) were diagnosed and treated for pathology causing an obstruction of the IM. Demographics and clinical data are shown in Table 1. Three patients had a cyst obstructing the IM. Three had an obstructive membrane, two had severe septal deviation, two had concheal hypertrophy causing obstruction, and two had a dacryolith. Seven of our patients were females; the mean age was 24 years (range 15-36). The pathology was diagnosed in the right eye in two cases and left eye in the remaining six. Only patient number 8 had a prior history of nasal trauma. In four patients $(50 \%)$, we recognized more than one pathology leading to obstruction of the IM. All patients were initially treated by endoscopic targeted surgery to resolve the obstruction at the IM. Septoplasty was performed on two patients with a coexisting nasal septal deviation. Four patients $(50 \%)$ were left with a silicone intubation at the end of surgery. The average follow-up duration was 35 months (range 7-57) in which all but two patients had complete resolution of ocular symptoms. The two patients with persistent NLDO and epiphora underwent endoscopic DCR with complete resolution of symptoms.

\section{Discussion}

We describe a series of eight patients presented with NLDO and epiphora due to a diverse set of pathologies effecting the IM. Three cysts, three obstructive membranes, two severe septal deviations, two concheal hypertrophies, and two dacryoliths. To date, no large series reported dacryoliths and membranes obstructing the IM and previous 


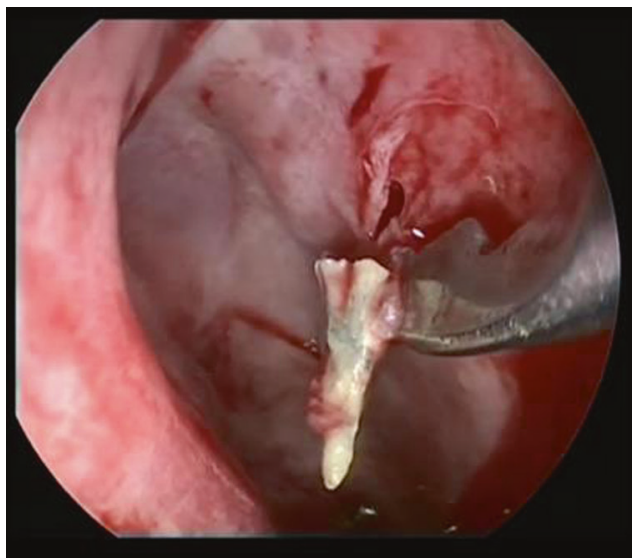

Fig. 1 Patient 1-A 22-year-old patient was referred to our clinic due to complaints of constant epiphora in the left eye and a history of dacryocystitis. Endoscopic DCR was scheduled, during which a mild swelling near the inferior meatus was noticed. Dilatation of Hasner's valve revealed a large lacrimal dacryolith. The dacryolith was extracted endoscopically and the DCR was aborted

reports of other IM pathologies were confined to case reports [3-8].

Previously published series suggest that nasal mucosa hypertrophy and septal deviation are common causes for NLDO [5, 6]. While these were common findings in our cases series too $(37.5 \%)$, we have found that it often coexists with other pathologies of the IM. Multiple pathologies might be accidental or have cause and effect relation between them. Failure to address both pathologies may leave the patient symptomatic as was suggested by Chan et al. [9].

Membranes obstructing Hasner's valve are the most common cause of primary congenital NLDO. More than $90 \%$ of the cases resolve spontaneously within the first year of life [10]. Nonetheless, we have found three such cases in adult patients (37.5\%). This finding is seldom found in adults and there are only a few similar reports in the literature. A question remains whether these obstructions represent a congenital malformation that become symptomatic later in life, or a secondary fibrotic process.

Similarly, nasolacrimal duct cysts and dacryoceles are considered congenital abnormalities of the NLD, and are usually diagnosed early in infancy. Such pathologies are possibly more common in adults than previously thought, as seen in our series and some other recent publications [3-7].

Dacryoliths (Fig. 1) are quite common in the lacrimal sac and are found in $5.7-18.3 \%$ of DCR surgeries [11]. Not many publications describe an obstruction of the IM by a dacyolith [5]. Risk factors for dacryolith formation include young age, female gender, smoking, and a history of dacryocystitis. Dacryoliths are composed of cellular derbies, proteins, calcium, ammonium, and other materials, and endoscopic extraction often brings instant relief and spares the need for any further surgical intervention as described in case number $1[8,12,13]$.

Tumors of the lacrimal sac and duct are rare but should not be overlooked. While the most common neoplasm of the lacrimal sac is squamous cell carcinoma, an obstruction of the IM by a hypertrophic lymphoid tissue has been previously described [5]. We have found no tumors in our series.

In this case series, patients were predominantly females with an average age significantly younger than in previously published case series [5, 6]. This could be a result of a selection bias in our clinic: young patients with NLDO were evaluated with a higher index of suspicion leading to a more meticulous evaluation of Hasner's valve.

Three patients had an incomplete obstruction, with only partial passage of irrigation with some degree of reflux. The assessment of the degree of reflux in those cases is problematic and inaccurate. Though some passage existed and the NLD was not completely obstructed, the clinical assessment along with the patients' signs and symptoms indicated surgical intervention.

Seven out of eight $(87 \%)$ patients in our series were referred for a computed tomography or magnetic resonance imaging (MRI) as part of their pre-operative evaluation. Although Golan et al. [2] have shown the importance of imaging in NLDO patients, it is not routinely preformed in our clinic, and it is reserved for atypical cases, usually in young patients.

Comparing patient 2 with patient 3 highlights the complexity of coexisting pathologies and the importance of imaging in selected cases. Both are young females with symptomatic epiphora. On imaging, both had a significant dacryocele and had successful endoscopic removal of an IM pathology: a cyst (patient 2) and a membrane (patient 3). Patient 3 remained symptomatic, possibly due to stenosis of the distal NLD that was noted in the pre-operative MRI: dilated NLD of patient 2 seen in Fig. 2 as opposed to the stenotic NLD of patient 3 seen in Fig. 3. Therefore, the patient was scheduled for an endoscopic DCR after which symptoms resolved completely. Integration of different modalities together with meticulous patient selection may decrease failure rates, by eliminating such cases where surgical manipulation of the IM may not suffice and DCR may be a preferable surgical approach.

A pre-operative endoscopic diagnosis of IM pathologies is preferred for optimal planning of surgical treatment. However, such examination should include medial concheal deflection and should be conducted under local anesthesia with de-congestion of the nasal cavity. Though recommended, it is especially difficult in young patients and not 
Fig. 2 Patient 2-A 15-year-old patient presented with left eye epiphora and a non-tender fullness in the lacrimal fossa. MRI coronal (a) and axial (b) MRI T2 images showing a left dcryocele and dilataion of left NLD. Endoscopic examination revealed a cyst obstructing Hasner's valve. Surgery was limited to excision of the cyst and dilatation of the inferior meatus orifice with complete resolution of symptoms during follow-up

Fig. 3 Patient 3-A 15-year-old patient presented with epiphora from the left eye and a non-tender fullness in the lacrimal fossa. MRI $\mathrm{T} 1$ coronal (a) and axial (b) section showing a dacryocele with a stenotic NLD. The patient went through endoscopic excision of a membrane obstructing the IM. Despite free passage of fluorescein at the end of surgery, the patient remained symptomatic and was referred for an endoscopic endonasal DCR with a complete resolution of symptoms
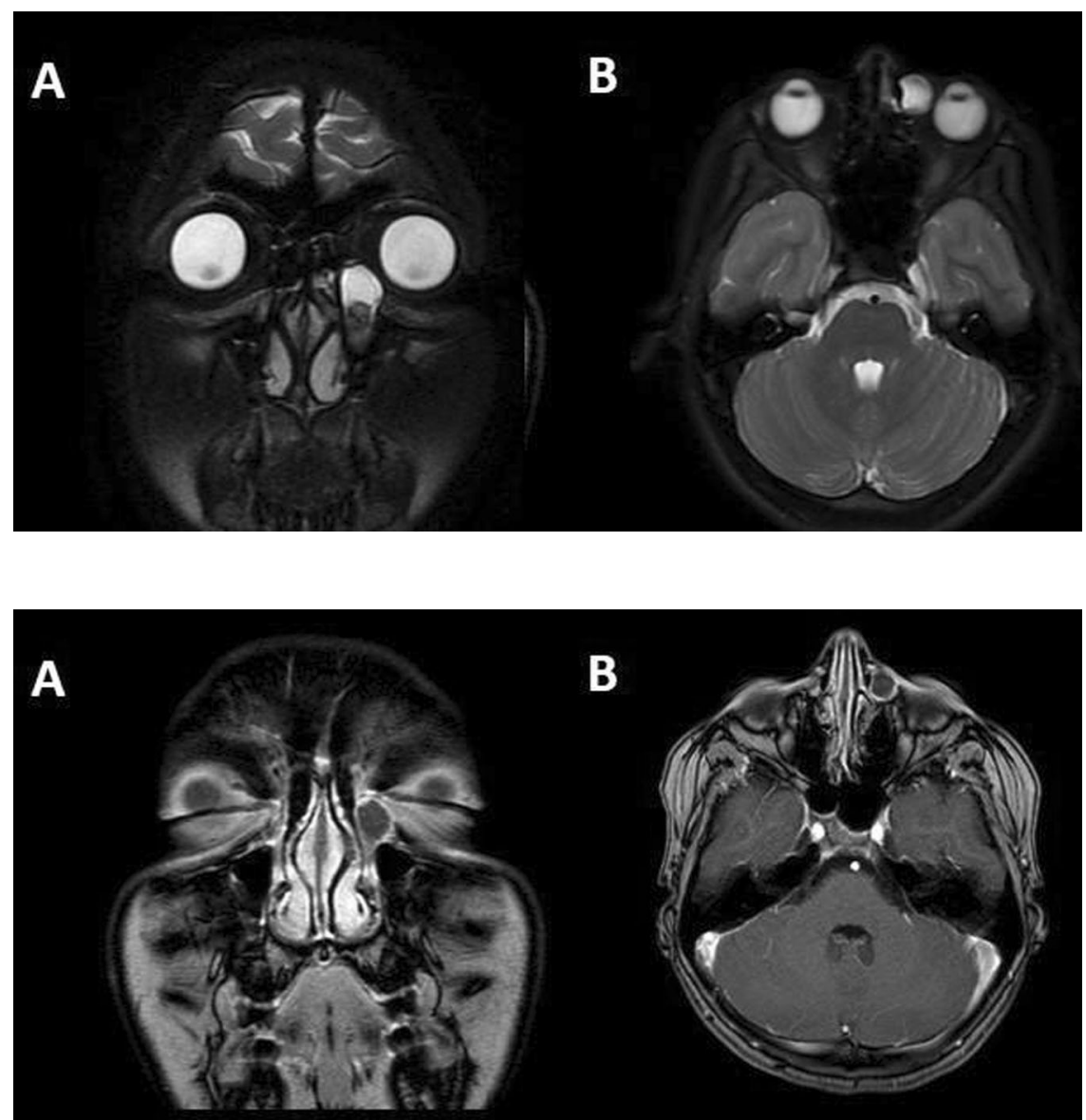

always possible in the clinic. In such cases a basic endoscopic examination of the nasal cavity should be initially performed, with a more profound endoscopic examination at the beginning of surgery including a meticulous examination of the IM.

The reported success rate of endoscopic DCR surgeries is 85-90\% [14-16]. Endoscopic treatment of the IM brought relief to six out of the eight reported patients (75\%), which represent $3.2 \%$ of the 186 DCR surgeries performed during the study's time frame. This number is similar to previously published data by Pedram et al. who found a success rate of about $70 \%$ in a similar case series [6]. A possible explanation may be in the finding of multiple pathologies affecting the NLD, in particular, septal deviation and inferior concheal hypertrophy, as were seen in three of our patients. Although all eight patients could have been managed successfully with standard DCR, targeted endoscopic management of the IM pathologies has only slightly less favorable success rate, possibly because of our small cohort, and it constitutes a less invasive procedure with fewer associated morbidities and less discomfort for the patient.

Due to the small number of patients in our series, it is not possible to assess the effect of silicone intubation in such cases. This may be the basis to future studies regarding intubation usage. In addition, although all cases described above were treated under general anesthesia, we believe that selected cases could have been done as an office procedure avoiding the costs and complications of general anesthesia.

\section{Conclusions}

A pre-operative and intra-operative endoscopic evaluation of the nasal cavity and IM as part of NLDO workup should be considered for patients intended to undergo endoscopic DCR, especially in young patients. Endoscopy not only serves as a diagnostic tool, but may also allow a DCR sparing targeted treatment.

\section{Summary}

\section{What was known before}

- Inferior meatus obstruction is an underdiagnosed cause of epiphora. 


\section{What this study adds}

- Multiple pathologies may co-exist in the same patient. In select cases of NLDO, diagnosis and treatment can be done endoscopically, avoiding the need for dacryocystorhinostomy.

\section{Compliance with ethical standards}

Conflict of interest The authors declare that they have no conflict of interest.

\section{References}

1. Ohtomo K, Ueta T, Toyama T, Nagahara M. Predisposing factors for primary acquired nasolacrimal duct obstruction. Graefes Arch Clin Exp Ophthalmol. 2013;251:1835-9.

2. Golan S, Leibovitch I, Landsberg R. Unexpected pathologies in patients referred for endoscopic DCR. Eur Arch Oto-RhinoLaryngol. 2014;271:3053-8.

3. Eloy P, Poirrier L, Nicoli T, et al. Cystic dilation of the distal end of the nasolacrimal duct: underrated cause of epiphora in adults and its endoscopic treatment. Rhinology. 2012; 50:436-41.

4. DelGaudio JM, Wojno T. Nasolacrimal duct orifice cysts in adults: a previously unrecognized, easily treatable cause of epiphora. Laryngoscope. 2007;117:1830-3.

5. Rogers GA, Murchison AP, Wojno TH, DelGaudio JM. Inferior meatus endoscopy and directed treatment for epiphora: early experience with a novel approach. Otolaryngol Head Neck Surg. 2009;140:579-84.

6. Pedram D, John M, DelGaudio M. Inferior meatus surgery for distal nasolacrimal duct obstructions long-term outcomes and treatment paradigm. JAMA Otolaryngol Head Neck Surg. 2014;140:736-41.

7. Koltsidopoulos P, Papageorgiou E, Konidaris VE, Skoulakis C. Idiopathic acquired dacryocystocele treated with endonasal endoscopic dacryocystorhinostomy. BMJ Case Rep. 2013; bcr2013200540.

8. Ben Cnaan R, Moosajee M, Heatley CJ, Olver JM. Endoscopic endonasal retrieval of a nasolacrimal duct stone via the valve of Hasner in the inferior meatus. Ophthalmic Plast Reconstr Surg. 2012;28:e48-e50.

9. Chan DM, Golubev I, Shipchandler TZ, Nunery WR, Lee HH. Improving outcomes by combining septoplasty with primary external dacryocystorhinostomy. Am J Otolaryngol Head Neck Med Surg. 2014;35:309-12.

10. Wallace EJ, Cox A, White P, MacEwen CJ. Endoscopic-assisted probing for congenital nasolacrimal duct obstruction. Eye (Lond). 2006;20:998-1003.

11. Yazici B, Hammad AM, Meyer DR. Lacrimal sac dacryoliths predictive factors and clinical characteristics. Ophthalmol. 2001;6420:1308-12.

12. Dhillon N, Kreis AJ, Madge SN. Dacryolith-induced acute dacryocystitis: a reversible cause of nasolacrimal duct obstruction. Orbit. 2014;33:199-201.

13. Seo ST, Park JS, Kim YM, Rha KS. A huge dacryolith presenting as a mass of the inferior meatus. Korean J Otorhinolaryngol-Head Neck Surg. 2016;59:238-41.

14. Marcet MM, Kuk AKT, Phelps PO. Evidence-based review of surgical practices in endoscopic endonasal dacryocystorhinostomy for primary acquired nasolacrimal duct obstruction and other new indications. Curr Opin Ophthalmol. 2014;25:443-8.

15. Liang J, Hur K, Merbs SL, Lane AP. Surgical and anatomic considerations in endoscopic revision of failed external dacryocystorhinostomy. Otolaryngol Head Neck Surg. 2014;150:901-5.

16. Huang J, Malek J, Chin D, et al. Systematic review and metaanalysis on outcomes for endoscopic versus external dacryocystorhinostomy. Orbit. 2014;33:81-90. 